Matthieu J. C. M. Rutten

James M. P. Collins

Bas J. Maresch

Jacques H. J. M. Smeets

Caroline M. M. Janssen

Lambertus A. L. M. Kiemeney

Gerrit J. Jager

\section{Glenohumeral joint injection: a comparative study of ultrasound and fluoroscopically guided techniques before MR arthrography}

Received: 30 June 2008

Accepted: 19 September 2008

Published online: 29 October 2008

(C) The Author(s) 2008

M. J. C. M. Rutten $(\bowtie)$ •

C. M. M. Janssen - G. J. Jager

Department of Radiology, Jeroen

Bosch Hospital,

Nieuwstraat 34,

$5211 \mathrm{NL}$

's-Hertogenbosch, The Netherlands

e-mail: m.rutten@jbz.nl

Tel.: +31-73-6992000

Fax: +31-73-6992601

e-mail: c.janssen@jbz.nl

e-mail: g.jager@jbz.nl

\section{J. M. P. Collins}

Department of Radiology,

Medical Centre Leeuwarden,

H. Dunantweg 2,

8934 AD

Leeuwarden, The Netherlands

e-mail: j.collins@znb.nl

\section{B. J. Maresch}

Department of Radiology, Hospital

Gelderse Vallei,

Willy Brandtlaan 10,

6716 RP Ede, The Netherlands

e-mail: mareschb@zgv.nl

\author{
J. H. J. M. Smeets \\ Department of Radiology, Slingeland \\ Hospital, \\ Kruisbergseweg 25, \\ 7009 BL Doetinchem, The Netherlands \\ e-mail: jsmeets@planet.nl
}

\section{A. L. M. Kiemeney}

Department of Epidemiology and

Biostatistics, Radboud University

Nijmegen Medical Centre,

Geert Grooteplein Noord 21,

P.O. Box 9101, $6500 \mathrm{HB}$

Nijmegen, The Netherlands

e-mail: b.kiemeney@ebh.umcn.nl

\begin{abstract}
To assess the variability in accuracy of contrast media introduction, leakage, required time and patient discomfort in four different centres, each using a different imageguided glenohumeral injection technique. Each centre included 25 consecutive patients. The ultrasoundguided anterior (USa) and posterior approach (USp), fluoroscopic-guided anterior (FLa) and posterior (FLp) approach were used. Number of injection attempts, effect of contrast leakage on diagnostic quality, and total room, radiologist and procedure
\end{abstract}

times were measured. Pain was documented with a visual analogue scale (VAS) pain score. Access to the joint was achieved in all patients. A successful first attempt significantly occurred more often with US (94\%) than with fluoroscopic guidance (72\%). Leakage of contrast medium did not cause interpretative difficulties. With US guidance mean room, procedure and radiologist times were significantly shorter $(\mathrm{p}<0.001)$. The USa approach was rated with the lowest pre- and post-injection VAS scores. The four image-guided injection techniques are successful in injection of contrast material into the glenohumeral joint. US-guided injections and especially the anterior approach are significantly less time consuming, more successful on the first attempt, cause less patient discomfort and obviate the need for radiation and iodine contrast.

Keywords Ultrasound .

Fluoroscopy $\cdot$ Shoulder · Imageguided injection $\cdot$ MR arthrography

\section{Introduction}

MR arthrography is frequently performed in the assessment of shoulder problems [1]. Intra-articular injection of a gadolinium chelate or a saline solution improves the detection of rotator cuff tears and demonstration of the labrum [1].
Deposition of contrast media into the glenohumeral joint may be a challenge for some radiologists. According to the numerous publications on this subject, it is still an item of investigation [2-20]. There are various techniques from blind $[4,17,19]$ to image guided $[2,3,10,12]$. Glenohumeral injections are generally performed using an anterior approach under fluoroscopic guidance, which 
was first described in 1933 by Oberholzer [21] and modified by Schneider [22]. Other methods for intraarticular injection of the glenohumeral joint have been described, e.g., fluoroscopically guided using a posterior approach $[9,10]$, palpation directed $[4,19]$, ultrasound (US) $[2,3,14]$, CT [11] and MR guided [7, 20]. These studies deal mostly with the techniques and/or their feasibility. Nowadays there is growing interest in evaluating and improving radiologists' interpretive performance and efficiency in delivering patient care. However, little is known about differences in accuracy of contrast injection, the effect of contrast leakage on image interpretation [19], the time taken and the discomfort experienced by the patient [23, 24] among different radiologists using different techniques.

The purpose of our study was to prospectively assess the variation in accuracy, effect of leakage of contrast medium on diagnostic quality, the time required and the discomfort experienced by the patient for the introduction of contrast material into the glenohumeral joint with four different frequently applied image-guided (fluoroscopy and US) injection techniques.

\section{Materials and methods}

Patients

A prospective study was performed in four centres each including 25 consecutive patients with shoulder instability who were referred for MR arthrography by orthopaedic surgeons. Patient characteristics are illustrated in Table 1. Patients who had previously undergone shoulder surgery were excluded. Institutional review board approval and verbal consent were obtained.

Four radiologists in four different centres were deliberately chosen because each routinely used a different approach for glenohumeral injection. In this way we were able to audit and compare the daily practice of various glenohumeral humeral injection techniques applied by experienced musculoskeletal radiologists ( $>9$ years of experience each). In each centre a different intra-articular approach was applied by an experi- enced musculoskeletal radiologist, fluoroscopic-guided anterior (FLa), fluoroscopic-guided posterior (FLp), US-guided anterior (USa) and US-guided posterior (USp).

All patients underwent intra-articular injection of gadoterate meglumine (gadoteric acid) $0.0025 \mathrm{mmol} / \mathrm{ml}$ (Artirem ${ }^{\circledR}$; Guerbet S.A., Villepinte, France), which is a dilution (1:200) of Dotarem ${ }^{\circledR}$ (Gd-DOTA) provided in prefilled syringes of $20 \mathrm{ml}$, until intra-articular pressure rose or up to a maximum of $20 \mathrm{ml}$.

\section{Injection techniques}

All radiologists used the technique and materials with which they were familiar. The FLp and Fla injections were performed with a $1.2 \mathrm{~mm} \times 88.9-\mathrm{mm}$ spinal needle $(18$ gauge, 3.5 inch) and a $0.9 \times 90-\mathrm{mm}$ spinal needle $(20$ gauge), respectively, and the US-guided injections with a $0.8 \times 50-\mathrm{mm}$ bevelled needle (21-gauge) without a stylet.

The Fla injections are performed with the patient supine in a straight anteroposterior position with the shoulder slightly externally rotated. The needle is directed vertically under fluoroscopic control at the junction of the middle and lower thirds of the medial part of the humeral head (Figs. 1 and 3).

See also Figs. 2 and 3.

The Flp injections are performed in prone position with the symptomatic shoulder slightly raised until the glenohumeral joint is seen tangentially $[9,10]$. The injection site is infiltrated with local anaesthetic [prilocaïnehydrochloride $10 \mathrm{mg} / \mathrm{ml}$ (Citanest ${ }^{\mathbb{R}} 1 \%$ ), Astra Pharmaceutica, Zoetermeer, The Netherlands] using a $0.8 \times 50-\mathrm{mm}$ bevelled needle (21 gauge). With the shoulder in a neutral position or slightly internally rotated, the needle is aimed at the inferomedial quadrant of the humeral head and advanced vertically under fluoroscopic guidance to the cartilage of the humeral head (Figs. 2 and 4) [9, 10].

The USa approach was performed according to Valls [3]. Patients are supine with the shoulder slightly externally rotated. A HDI 5000 SonoCT (ATL/Philips, Best, The Netherlands) with a 5-8-MHz curved array transducer was used to visualise the needle (Fig. 5A). After skin and transducer preparation with alcohol $70 \%$, the patient's

Table 1 Four patient groups

\begin{tabular}{|c|c|c|c|c|c|c|}
\hline & \multirow{2}{*}{$\begin{array}{l}\text { Patients } \\
\text { No. }\end{array}$} & \multicolumn{2}{|c|}{ Gender } & \multirow{2}{*}{$\begin{array}{l}\text { Age } \\
\text { Mean/median (range) }\end{array}$} & \multicolumn{2}{|c|}{ Sidedness } \\
\hline & & $\mathrm{M}$ & $\mathrm{F}$ & & $\mathrm{L}$ & $\mathrm{R}$ \\
\hline FLa & 25 & 17 & 7 & 39/44 (14-69) & 9 & 16 \\
\hline FLp & 25 & 14 & 11 & $44 / 44(28-62)$ & 14 & 11 \\
\hline USa & 25 & 16 & 9 & 35/34 (18-59) & 9 & 16 \\
\hline USp & 25 & 19 & 6 & $36 / 37(16-69)$ & 10 & 15 \\
\hline
\end{tabular}

Number of patients per group, gender, age and sidedness of the punctured shoulders are displayed

USa: US-guided anterior injection, USp: US-guided posterior injection, FLa: fluoroscopic-guided anterior injection, FLp: fluoroscopicguided posterior injection 


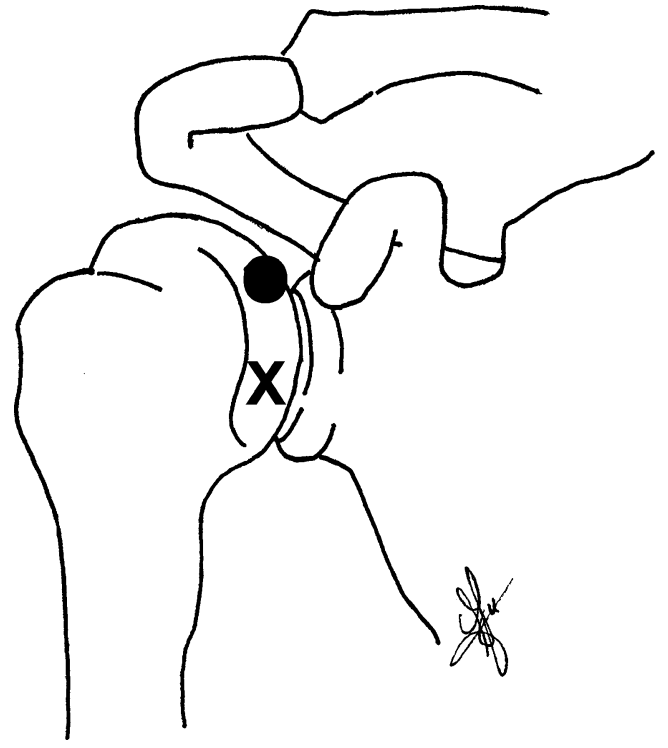

Fig. 1 Schematic drawing of the anterior view of the right shoulder showing the puncture sites of the fluoroscopic- (X) and US-guided $(\bullet)$ anterior injection techniques

shoulder was draped in sterile fashion. The needle is inserted at the level of the coracoid, from lateral to medial, aimed at the medial border of the humeral head (Figs. 1 and 5) [3]. The contrast medium can be seen flowing in the direction of the subscapular recess and joint space.

The USp approach, described by Cicak [2] and modified by Zwar [14], was performed using a APLIO US machine (Toshiba Medical Systems Corporation, Tokyo, Japan) with a 7.5-14-MHz linear array transducer (Toshiba PLF-

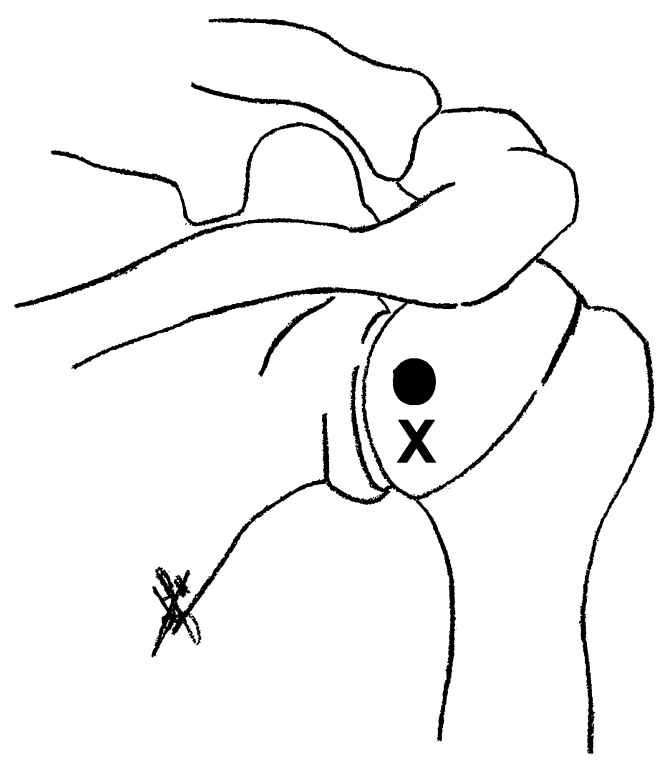

Fig. 2 Schematic drawing of the posterior view of the right shoulder showing the puncture sites of the fluoroscopic- (X) and US-guided $(\bullet)$ posterior injection techniques

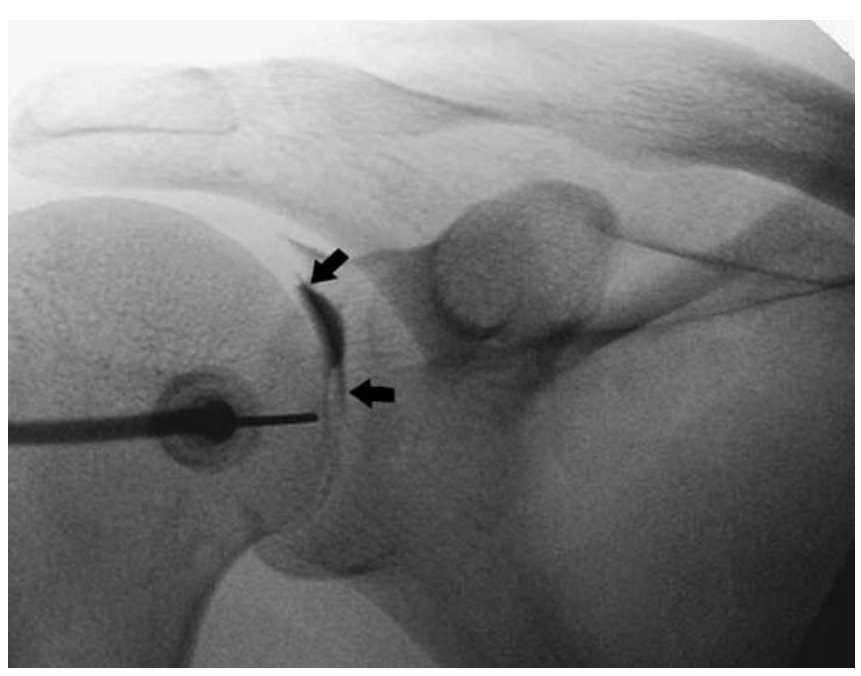

Fig. 3 Fluoroscopic-guided anterior approach. X-ray of the anterior view of a right-hand shoulder showing the needle entry site. The needle is inserted vertically at the inferomedial quadrant of the humeral head. The intra-articular position of the needle tip is confirmed by injecting a little iodinated contrast media, which demarcates the hyaline cartilage (black arrows) of the humeral head

$805 \mathrm{ST})$. The patient is either lying obliquely prone on the contralateral shoulder or sitting upright with the back to the radiologist and the ipsilateral hand on the contralateral shoulder. After skin and transducer preparation with alcohol $70 \%$, the patient's shoulder was draped in sterile fashion. The needle is inserted, from lateral to medial, parallel to the long axis of the transducer and advanced under US control in the joint between the humeral head and the posterior glenoid labrum (Figs. 2 and 6) [14].

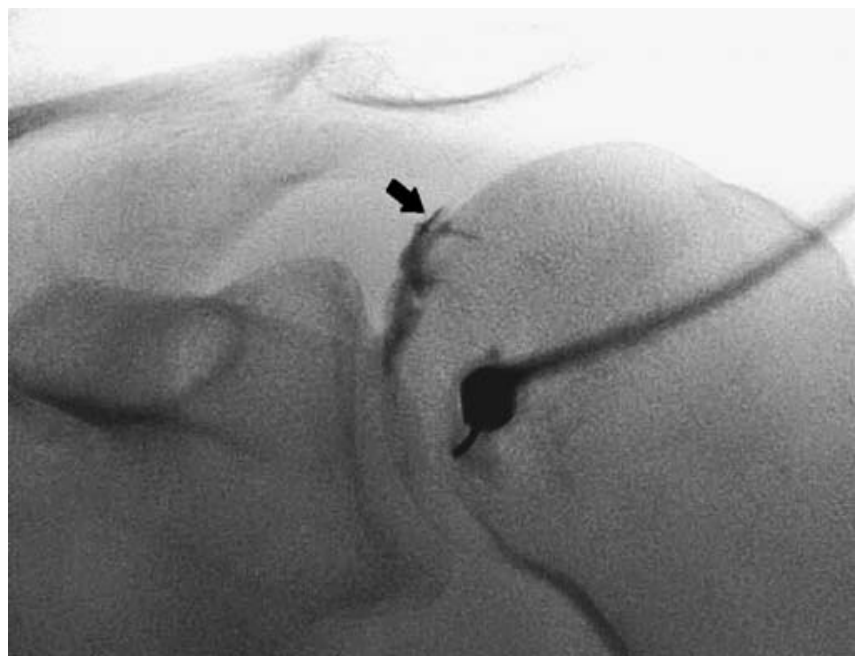

Fig. 4 Fluoroscopic-guided posterior approach. X-ray of the posterior view of a right-hand shoulder showing the needle entry site. The needle is inserted vertically at the inferomedial quadrant of the humeral head. The intra-articular position of the needle tip is confirmed by injecting a little iodinated contrast media, which demarcates the hyaline cartilage (black arrow) of the humeral head 
Fig. 5 US-guided anterior approach. a Sonogram of a righthand shoulder showing the needle track (arrows) from lateral to medial with the USa approach. The needle is inserted at the level of the coracoid (C). The tip of the needle is in intra-articular position with the tip underneath the subscapular tendon (SSC) and bordering the humeral head $(\mathrm{H})$. b Corresponding cadaver section showing the optimal needle track (white line)

Time assessment

To be able to monitor the time taken for the procedure in four different centres, with different personel, a simple method of scoring how things were done was standardised.

Room time (time between the patient entering and leaving the room), radiologist time (time between entering and leaving the room) and procedure time (time between starting and ending the injection procedure) were recorded. Mean times were assessed, and because of the maximum time of Fla and Flp, we also assessed the median times (Table 3).
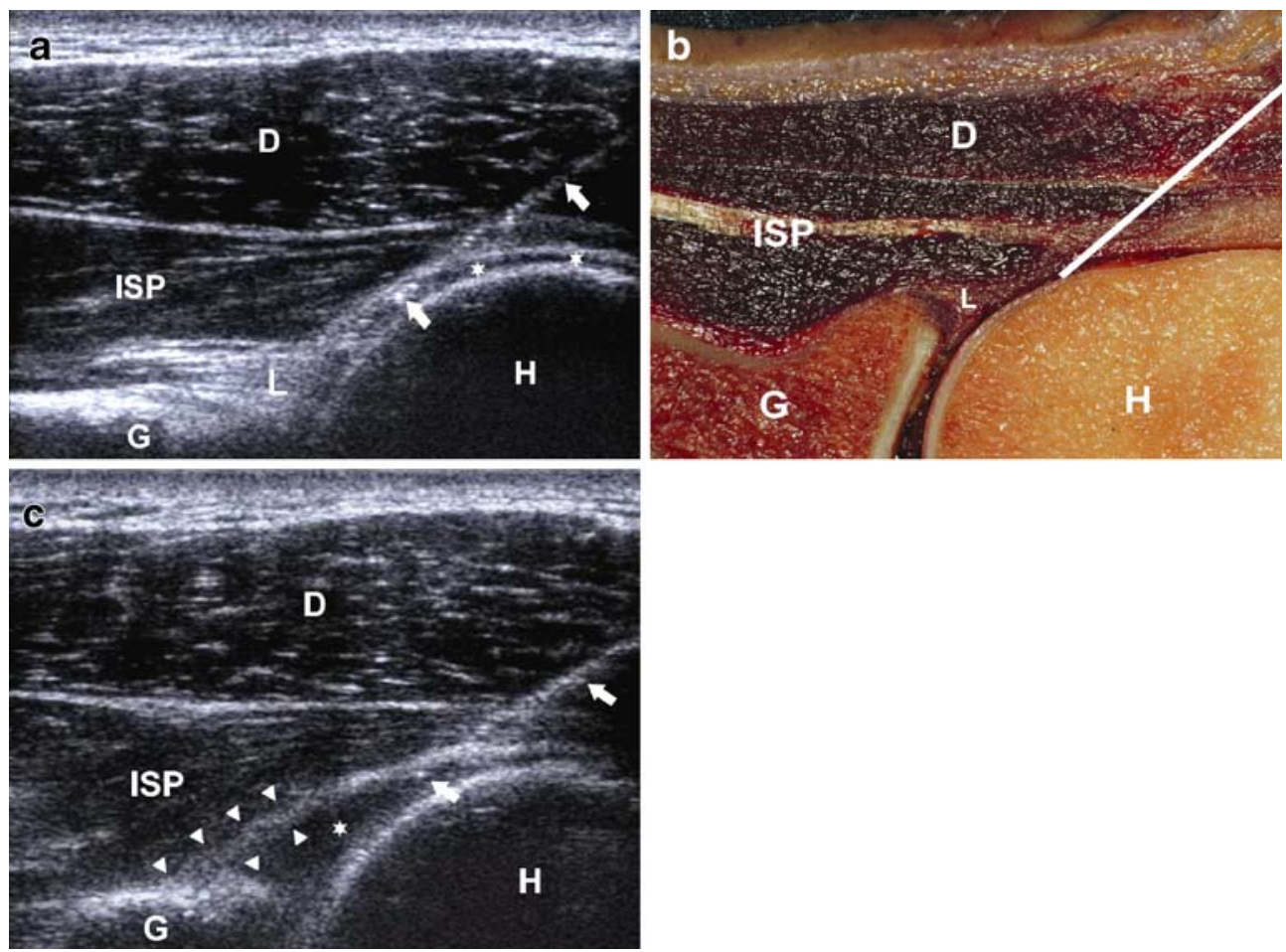

Fig. 6 US-guided posterior approach. a Sonogram of a right-hand shoulder showing the needle track (arrows) from lateral to medial with the USp approach. The needle is inserted at the midlevel of the humeral head $(\mathrm{H})$. The needle is in intra-articular position with the tip underneath the infraspinatus tendon (ISP) and posterior labrum (L) and bordering the hyaline cartilage (asterisks) of the humeral head. G: glenoid. b Corresponding cadaver section showing the
Evaluation of intra-articular administration of contrast material

The number of attempts needed to enter the joint space was recorded. An attempt to inject a joint was defined as the need for repositioning of the needle after unsuccessful test injection.

The joint space was considered to be entered when the needle contacted the humeral head cartilage, test injection was performed without resistance and the intra-articular position of contrast material was confirmed with fluoroscopy by injection of 1-2 $\mathrm{ml}$ iodinated nonionic contrast (Omnipaque 300; Amersham Cygne, Eindhoven, The

optimal needle track (white line). c Sonogram following injection of $15 \mathrm{ml}$ injected gadoterate meglumine (Artirem $\left.{ }^{\circledR}\right)$ (asterisk). The correct intra-articular position of the needle (arrows) can be visualised real-time during injection, but is also confirmed by the 'comma'-like configuration of the posterior labrum (arrowheads), which is lifted by the intra-articularly injected fluid (asterisk). G: glenoid, H: humearal head, ISP: infraspinatus tendon 
Netherlands). Confirmation with real time US can be performed without the use of iodinated contrast media by visualising the intra-articular flow of fluid during injection or by visualising the intra-articularly injected fluid underneath the capsule and/or labrum posteriorly and underneath the subscapular tendon anteriorly, or around the long head of the biceps tendon.

The amount of injected contrast material was recorded.

MR imaging was performed within $15 \mathrm{~min}$ of the injection with a 1.0-T or a 1.5-T MR system. In all centres a dedicated receive-only shoulder coil was used. Images were used for diagnostic purpose and to assess retrospectively the intraarticular position and contrast leakage by an independent blinded radiologist who did not perform the procedure. Leakage was graded using a five-grade scale (Table 2).

Visual analogue scale

Before and after injection, patients graded their anticipated and experienced level of pain. A visual analogue scale (VAS) was used, with a scale from 0 ("no pain") to 100 ("unbearable pain"). Complications were recorded.

\section{Statistical analysis}

For descriptive purposes, mean and standard deviations are reported for room time, radiologist time, total procedure time and VAS scores. Because the variables were non-normally distributed, nonparametric KruskalWallis tests were used to test for statistical differences. Statistical significance was accepted if the $P$ value was less than 0.05 . The comparisons between the techniques were not adjusted for the patients' sex, age or right or left shoulder. The distribution of all categorical variables was presented in frequencies. Differences in these distributions were tested for statistical significance using chi-square tests.

All statistical analyses were performed using SPSS, version 14.0.2 for Windows (Chicago, IL) by an experienced statistician (L.K.).

\section{Results}

\section{Patient groups}

As illustrated in Table 1, the patient groups involved in this study were quite similar in sex, age distribution and sidedness of the affected shoulder. There was a slight predominance in right shoulders, as one might expect, as most people are right-handed. Patients' gender and left or right shoulder were not significantly related to room time, radiologist time, total procedure time and difference between the pre- and post-procedure VAS scores. The age of the patients was only weakly $\left(\mathrm{r}^{2}<0.04\right)$ correlated with the three time variables and not at all correlated with the VAS score difference. Therefore, the analyses were not adjusted for age, sex and laterality.

Table 2 Accuracy, number of attempts to gain access to the glenohumeral joint, injected volume and leakage of contrast media with four different image-guided approaches

\begin{tabular}{|c|c|c|c|c|c|c|c|}
\hline Number of patients (N) & $\begin{array}{l}\text { Overall } \\
100\end{array}$ & $\begin{array}{l}\text { FLa } \\
25\end{array}$ & $\begin{array}{l}\text { FLp } \\
25\end{array}$ & $\begin{array}{l}\text { USa } \\
25\end{array}$ & $\begin{array}{l}\text { USp } \\
25\end{array}$ & $\begin{array}{l}\mathrm{FL}(\mathrm{a}+\mathrm{p}) \\
50\end{array}$ & $\begin{array}{l}\mathrm{US}(\mathrm{a}+\mathrm{p}) \\
50\end{array}$ \\
\hline Intra-articular injection & $100(100 \%)$ & $25(100 \%)$ & $25(100 \%)$ & $25(100 \%)$ & $25(100 \%)$ & $50(100 \%)$ & $50(100 \%)$ \\
\hline 1st attempt & $83(83 \%)$ & $19(76 \%)$ & $17(68 \%)$ & $24(96 \%)$ & $23(92 \%)$ & $36(72 \%)$ & $47(94 \%)$ \\
\hline 2nd attempt & $14(14 \%)$ & $5(20 \%)$ & $6(24 \%)$ & $1(4 \%)$ & $2(8 \%)$ & $11(22 \%)$ & $3(6 \%)$ \\
\hline 3rd attempt & $3(3 \%)$ & $1(4 \%)$ & $2(8 \%)$ & $0(0 \%)$ & $0(\%)$ & $3(6 \%)$ & $0(0 \%)$ \\
\hline No leakage & $49(49 \%)$ & $8(32 \%)$ & $13(52 \%)$ & $16(64 \%)$ & $12(48 \%)$ & $21(42 \%)$ & $28(56 \%)$ \\
\hline Minimal leakage noncompromising & $24(24 \%)$ & $12(48 \%)$ & $3(12 \%)$ & $4(16 \%)$ & $5(20 \%)$ & $15(30 \%)$ & $9(18 \%)$ \\
\hline Massive leakage noncompromising & $27(27 \%)$ & $5(20 \%)$ & $9(36 \%)$ & $5(20 \%)$ & $8(32 \%)$ & $14(28 \%)$ & $13(26 \%)$ \\
\hline Minimal leakage compromising & $0(0 \%)$ & $0(0 \%)$ & $0(0 \%)$ & $0(0 \%)$ & $0(0 \%)$ & $0(0 \%)$ & $0(0 \%)$ \\
\hline Massive leakage compromising & $0(0 \%)$ & $0(0 \%)$ & $0(0 \%)$ & $0(0 \%)$ & $0(0 \%)$ & $0(0 \%)$ & $0(0 \%)$ \\
\hline Minimal injected volume (ml) & 5.0 & 10.0 & 9.0 & 5.0 & 10.0 & 9.0 & 5.0 \\
\hline Maximal injected volume (ml) & 20.0 & 20.0 & 20.0 & 20.0 & 20.0 & 20.0 & 20.0 \\
\hline Mean injected volume (ml) & 15.6 & 14.5 & 14.9 & 14.6 & 18.6 & 14.7 & 16.6 \\
\hline Local anaesthetics & - & No & Yes & No & No & - & - \\
\hline Needle size (gauge) & - & 20 & 18 & 21 & 21 & - & - \\
\hline
\end{tabular}

USa: US-guided anterior injection, USp: US-guided posterior injection, FLa: fluoroscopic-guided anterior injection, FLp: fluoroscopicguided posterior injection, FL $(\mathrm{a}+\mathrm{p})$ : fluoroscopic-guided anterior and posterior approach, US $(\mathrm{a}+\mathrm{p})$ : US-guided anterior and posterior approach 


\section{Accuracy}

Injection of the glenohumeral joint was successfully performed in all 100 patients enrolled in this study (Table 2). Overall access to the joint was gained on the first attempt in $83(83 \%)$ patients, on the second attempt in $14(14 \%)$ patients and on the third attempt in $3(3 \%)$ patients. For fluoroscopic or US guidance the first attempt scores were $72 \%$ and $94 \%$, respectively. With fluoroscopy a second and third attempt was needed in $22 \%$ and $6 \%$, respectively, whereas with US guidance a second attempt in only $6 \%$, and a third attempt was not needed in any of the cases (Table 2).

The differences between the techniques in the frequency of more than one attempt needed were statistically significant (chi-square test $\mathrm{df}=3: \mathrm{p}=0.026$ ).

No complications of the vital structures, such as vessels, nerves and tendons, were encountered clinically and with MR imaging. Four (4\%) patients had a vasovagal reaction, two patients in the USp group and two patients in the FLa group. No further complications were reported in any of the groups as, for example, bleeding at the injection site, swelling or compromise of movements.

\section{Contrast material leakage}

The various injection techniques were qualitatively comparable with regard to extra-articular leakage of contrast material. In $51 \%$ of all procedures minimal (24\%) and massive (27\%) leakage occurred, which did not hamper in any way the evaluation of the MR images. Minimal leakage was most frequent in the FLa approach $(48 \%)$ and massive leakage in both posterior approaches (FLp: 36\% and USp: 32\%) (Table 2).
The differences in non-compromising leakage were statistically significant (chi-square test, $\mathrm{df}=6: \mathrm{p}=0.045$ ).

Time assessment

Fluoroscopy and US room times, overall procedure times and radiologist's times are summarised in Table 3 as minimum - maximum times (mean).

The mean room times for US guidance were significantly shorter when compared to the time needed using fluoroscopic guidance (Table 3). The shortest mean procedure time required was noted for the USa approach $(1: 34 \mathrm{~min})$. The longest mean procedure time noted was that using the FLp approach (9:48 $\mathrm{min})$. Also noteworthy is the difference in mean procedure time between the FLa (4:09 $\mathrm{min}$ ) and FLp approach (9:48 min). The minimum procedure times were similar among all four approaches; however, the maximum procedure times were significantly shorter using US guidance (Table 3 ).

On average the procedure under US guidance was shorter than under fluoroscopic guidance, using significantly less $(>50 \%)$ room time and radiologist time (Table 3).

\section{Discomfort}

The VAS score obtained before and after shoulder injection is summarised in Table 4. The VAS scores before the procedure varied among the four different procedures, but not significantly so (Kruskal-Wallis $p=0.108$ ). The variation in the VAS scores post-procedure was a little bit larger and statistically significant (Kruskal-Wallis $p=0.023$ ). In three of the four groups there was a general tendency for

Table 3 Required time (minutes) for US- and fluoroscopic-guided glenohumeral injection

\begin{tabular}{|c|c|c|c|}
\hline & $\begin{array}{l}\mathrm{T}_{\mathrm{Room}} \\
\text { Min - max (mean/median })\end{array}$ & $\begin{array}{l}\mathrm{T}_{\text {Procedure }} \\
\text { Min - Max (mean/median) }\end{array}$ & $\begin{array}{l}\mathrm{T}_{\text {Radiologist }} \\
\text { Min - max (mean/median })\end{array}$ \\
\hline FLa & 07:00 - 33:00 (17:03/17:00) & $00: 30-20: 00(04: 09 / 11: 43)$ & 03:00 - 27:00 (09:50/08:00) \\
\hline FLp & 12:00 - 29:00 (20:05/20:00) & $00: 45$ - 23:00 (09:48/09:00) & $10: 00-27: 00(17: 58 / 19: 00)$ \\
\hline USa & $06: 00-15: 00(09: 54 / 10: 00)$ & $00: 30-06: 00(01: 34 / 01: 00)$ & 04:00 - 13:15 (06:19/06:00) \\
\hline USp & 05:00 - 16:00 (09:18/09:00) & $00: 30-07: 30(03: 24 / 03: 00)$ & $01: 56-12: 00(05: 48 / 05: 00)$ \\
\hline $\mathbf{F L}(\mathbf{a}+\mathbf{p})$ & 07:00 - 33:00 (18:36/18:00) & $00: 30-23: 00(06: 58 / 07: 00)$ & 03:00 - 27:00 (13:54/15:00) \\
\hline $\mathbf{U S}(\mathbf{a}+\mathbf{p})$ & 05:00 - 16:00 (09:36/09:00) & $00: 30-07: 30(02: 29 / 02: 00)$ & $01: 56-13: 15(06: 03 / 06: 00)$ \\
\hline $\mathrm{T}_{\mathrm{US}}: \mathrm{T}_{\mathrm{FL}}$ & $71 \%-48 \%(52 \%)$ & $100 \%-33 \%(36 \%)$ & $64 \%-49 \%(44 \%)$ \\
\hline Kruskal-Wallis & $\mathrm{p}<0.001$ & $\mathrm{p}<0.001$ & $\mathrm{p}<0.001$ \\
\hline
\end{tabular}

$\mathrm{T}_{\text {Room }}=$ time that the room is occupied by patient. $\mathrm{T}_{\text {Procedure }}=$ time required for injection ( skin to skin), $\mathrm{T}_{\text {Radiologist }}=$ overall time radiologist in the US or fluoroscopy room

USa: US-guided anterior injection, USp: US-guided posterior injection, FLa: fluoroscopic-guided anterior injection, FLp: fluoroscopicguided posterior injection. FL $(\mathrm{a}+\mathrm{p})$ : fluoroscopic-guided anterior and posterior injection, US $(\mathrm{a}+\mathrm{p})$ : US-guided anterior and posterior injection, $\mathrm{T}_{\mathrm{US}}: \mathrm{T}_{\mathrm{FL}}$ : percentage of needed room, procedure and radiologist time with US guidance in relation to the time span for fluoroscopic guidance 
Table 4 VAS pain score for US- and fluoroscopy-guided glenohumeral injections

\begin{tabular}{|c|c|c|c|c|c|}
\hline \multirow{2}{*}{$\begin{array}{l}\text { Pre-procedure } \\
\text { Min-max (mean) }\end{array}$} & \multirow{2}{*}{$\begin{array}{l}\text { Post-procedure } \\
\text { Min-max (mean) }\end{array}$} & \multicolumn{4}{|c|}{ VAS difference } \\
\hline & & Mean & $\begin{array}{l}\text { Decrease } \\
\text { No. of patients }\end{array}$ & $\begin{array}{l}\text { Increase } \\
\text { No. of patients }\end{array}$ & $\begin{array}{l}\text { Equal } \\
\text { No. of patients }\end{array}$ \\
\hline FLa 0 - 90 (30) & $0-100$ & +9 & $3(12 \%)$ & $15(60 \%)$ & $7(28 \%)$ \\
\hline FLp $3-100$ & $0-100(28)$ & -14 & $15(60 \%)$ & $4(16 \%)$ & $6(24 \%)$ \\
\hline USa 0 - 75 (27) & $0-70(16)$ & -11 & $13(52 \%)$ & $4(16 \%)$ & $8(32 \%)$ \\
\hline USp $0-80$ (38) & $0-80$ & -11 & $13(52 \%)$ & $5(20 \%)$ & $7(28 \%)$ \\
\hline
\end{tabular}

USa: US-guided anterior injection, USp: US-guided posterior injection, FLa: fluoroscopic-guided anterior injection, FLp: fluoroscopicguided posterior injection, VAS-difference: VAS score post-procedure - VAS score pre-procedure

patients to indicate that the procedure was less painful than they had expected. In 44 patients the VAS score decreased following injection, whereas in 28 patients it increased, and in 28 patients the VAS score measured pre- and postprocedure remained equal. The mean VAS score of the FLp, USa and USp injections decreased 14, 11 and 11 points following injection, respectively, while it increased with 9 points in the FLa group (Kruskal-Wallis $p=0.001$ ). In fact, the post-procedure VAS score of the FLa procedure increased in $60 \%$ of the patients. There was a higher average pain score after FLa injection as compared to FLp. Patients who underwent US-guided procedures reported less pain than those who underwent the fluoroscopicguided procedures. The USa approach was rated with the lowest pre- and post-injection VAS scores.

\section{Discussion}

The main advantage of image-guided glenohumeral joint injection over blind injection is that the needle position can be confirmed and injection of contrast medium can be controlled. This is especially advantageous in teaching hospitals (when training residents) and when the number of MR arthrographies are limited.

The current study describes four different image-guided approaches. Intra-articular deposition of contrast material was successful in all patients, which is comparable with reported results in the literature $[2,3,10,13]$. The success rate of the first attempt with US guidance $(97 \%)$ was significantly better compared to fluoroscopic guidance $(72 \%)$.

In the literature the accuracy of "blind" glenohumeral injections varies from $26-100 \%$ [15-19]. Catalano et al. [19]] showed that with a palpation-directed posterior approach, the glenohumeral joint could be entered successfully on respectively the first, second and third attempt in $85 \%, 13 \%$ and $2 \%$ of the cases [19].

In this study the US procedure was less time consuming than the fluoroscopic-guided procedure $(2.30 \mathrm{~min}$ versus 7 min, i.e., only $36 \%$ of the time needed with fluoroscopic guidance). This may be due to the received pre-procedural local anaesthetics and the injection with iodinated contrast to confirm the intra-articular position. The reported time of blind injection by Catalano was $3 \mathrm{~min}$ [19]. The occupancy of the US room is only $52 \%$ of the fluoroscopy room (Table 3).

The figures of leakages are summarised in Table 2. The number is high compared to those reported by Catalano et al. [19]. This may be due to strict criteria. Every small amount of contrast material along the needle tract was graded minimal leakage. The frequent leakage did not cause interpretative difficulties. All patients were clinically suspected of having anterior labral problems, and therefore posterior leakage did not hamper interpretation. Contrast material anteriorly did not compromise diagnostic quality, because the contrast medium was usually depicted in close relationship to the needle tract. In six cases massive leakage was obviously due to capsular disruption $(n=3)$, pathology of the labrum $(n=2)$ and a full-thickness rotator cuff tear $(n=1)$.

Patients who undergo arthrography in general experience less pain than expected [23]. In our study $44 \%$ of the patients considered discomfort less than expected and $28 \%$ more than expected. However, in the subgroup of patients who underwent the FLa approach (Table 4), 60\% experienced more discomfort than expected. This can be explained by the fact that no local anaesthetics and a thicker needle (20 gauge) were used and the fact that manipulation of the stylet and syringe causes unintended movements of the needle.

In this study four minor complications, e.g., vasovagal reactions, were reported, which resolved without medical intervention: two in the USp group and two in the FLa group. Other minor complications included pain, urticaria and headaches [25]. All four procedures were difficult due to adipositas $(n=3)$ and postoperative status $(n=1)$. Hugo [25] showed in a multi-institutional survey a total complication rate of $3.6 \%$. Serious complications account for $0.02 \%$ and consist mainly of infections and rarely of anaphylaxis and vascular complications [25].

In our study there were no advantages comparing the anterior approach with the posterior approach. Some authors $[2,9,10,14,19]$ advocate the posterior approach, because most pathologies of the stabilising structures of the 
glenohumeral joint are encountered on the anterior aspect of the joint. Chung [9] shows that if a standard anterior fluoroscopic approach is used, the needle may traverse the primary stabilisers. To overcome this drawback a modified anterior approach through the rotator interval can be performed [13]. This is comparable with the US-guided anterior approach. With this approach the needle entry site is at the level between the coracoid process and the superior medial quadrant of the humeral head, which is cranial to the stabilising structures of the shoulder. This limits the risk of compromising diagnostic quality because of leakage of contrast material.

This study was not a strict comparative study between US and fluoroscopic techniques because all radiologists kept their own routine, introducing variables like needle size and the use of local anaesthetics. Also the differences in injection skills may play a role. Nevertheless, an important observation of this study is the wide variation in delivered patient care. Although this study is too small to analyse the contribution of different factors to this variation, it is important for physicians to be aware of these phenomena.

The results suggest that US-guided glenohumeral joint injection is preferable to fluoroscopic-guided glenohumeral joint injection. To confirm the findings a prospective study without variables such as needle size and local anaesthetics is needed. Furthermore, the number of operators should be extended to minimise the effect of differences in operator skills.

\section{Conclusion}

The four image-guided injection techniques are successful in injection of contrast material into the glenohumeral joint. Although the effect of other variables, such as size of the injection needle and the use of local anaesthetics, needs to be investigated, we encourage US-guided injections and especially the anterior approach, because in this study it was significantly less time consuming, more successful on the first attempt, can be performed with less patient discomfort and obviates the need for radiation and iodine contrast.

Acknowledgment The authors gratefully thank Stephan Muurling for his dedicated contribution in preparing the drawings in this article.

Open Access This article is distributed under the terms of the Creative Commons Attribution Noncommercial License which permits any noncommercial use, distribution, and reproduction in any medium, provided the original author(s) and source are credited.

\section{References}

1. Jbara M, Chen Q, Marten P, Morcos M, Beltran J (2005) Shoulder MR arthrography: how, why, when. Radiol Clin North Am 43:683-692

2. Cicak N, Matasovic T, Bajraktarevic T (1992) Ultrasonographic guidance of needle placement for shoulder arthrography. J Ultrasound Med 11:135-137

3. Valls R, Melloni P (1997) Sonographic guidance of needle position for MR arthrography of the shoulder. AJR 169:845-847

4. DeMouy EH, Menendez CV Jr, Bodin CJ (1997) Palpation-directed (nonfluoroscopically guided) salineenhanced MR arthrography of the shoulder. AJR 169:229-231

5. Lewin JS, Petersilge CA, Hatem SF et al (1998) Interactive MR imagingguided biopsy and aspiration with a modified clinical C-arm system. AJR 170:1593-1601
6. Trattnig S, Breitenseher M, Rand T et al (1999) MR imaging-guided MR arthrography of the shoulder: clinical experience on a conventional closed high-field system. AJR 172:1572-1574

7. Hilfiker PR, Weishaupt D, Schmid M, Dubno B, Hodler J, Debatin JF (1999) Real-time MR-guided joint puncture and arthrography. Eur Radiol 9:201-204

8. Miller TT (2000) MR arthrography of the shoulder and hip after fluoroscopic landmarking. Skeletal Radiol 29:81-84

9. Chung CB, Dwek JR, Feng S, Resnick D (2001) MR arthrography of the glenohumeral joint: A tailored approach. AJR 177:217-219

10. Farmer KD, Hughes PM (2002) MR arthrography of the shoulder: fluoroscopically guided technique using a posterior approach. AJR 178:433-434

11. Binkert CA, Verdun FR, Zanetti M, Pfirrmann CW, Hodler J (2003) CT arthrography of the glenohumeral joint: CT fluoroscopy versus conventional CT and fluoroscopy. Comparison of image-guidance techniques. Radiology 229:153-158
12. Jacobson JA, Lin J, Jamadar DA, Hayes CW (2003) Aids to successful shoulder arthrography performed with a fluoroscopically guided anterior approach. Radiographics 23:373-379

13. Dépelteau H, Bureau NJ, Cardinal E, Aubin B, Brassard P (2004) Arthrography of the shoulder: a simple fluoroscopically guided approach for targeting the rotator cuff interval. AJR 182:329-332

14. Zwar RB, Read JW, Noakes JB (2004) Sonographically guided glenohumeral joint injection. AJR 183:48-50

15. Sehti PM, Kingston S, Elattrache N (2005) Accuracy of anterior intraarticular injection of the glenohumeral joint. Arthroscopy 21:77-80

16. Berna-Serna JD, Redondo MV, Martinez F et al (2006) A simple technique for shoulder arthrography. Acta Radiol 47:725-729

17. Sehti PM, El Attrache N (2006) Accuracy of intra-articular injection of the glenohumeral joint: a cadaveric study. Orthopedics 29:149-152 
18. Hanchard N, Shanahan DHT, Thompsom J, Goodchild L (2006) Accuracy and dispersal of subacromial and glenohumeral injections in cadavers. J Rheumatol 33:1143-1146

19. Catalano OA, Manfredi R, Vanzulli A et al (2007) MR arthrography of the glenohumeral joint: modified posterior approach without imaging guidance. Radiology 242:550-554
20. Petersilge CA, Lewin JS, Duerk JL, Hatem SF (1997) MR arthrography of the shoulder: rethinking traditional imaging procedures to meet the technical requirements of MR imaging guidance. AJR 169:1453-1457

21. Oberholzer J (1933) Die Arthropneumoradiographie bei Habitueller Shulterluxation. Röntgenpraxis 5:589-590

22. Schneider R, Ghelman B, Kaye JJ (1975) A simplified injection technique for shoulder arthrography. Radiology 114:738-739
23. Robbins MI, Anzilotti KFJ, Katz LD, Lange RC (2000) Patient perception of magnetic resonance arthrography. Skeletal Radiol 29:265-269

24. Binkert CA, Zanetti M, Hodler J (2001) Patient's assessment of discomfort during MR arthrography of the shoulder. Radiology 221:775-778

25. Hugo PC, Newberg AH, Newman JS, Wetzner SM (1998) Complications of Arthrography. Semin Musculoskelet Radiol 2:345-348 